\title{
On the Thermodynamics of the Hopfield Neural Network with External Field
}

\author{
J. ULACZYK ${ }^{a}$, R.A. KosińSKI ${ }^{a, b}$ AND A. ZAGÓRSKI ${ }^{a}$ \\ ${ }^{a}$ Faculty of Physics, Warsaw University of Technology \\ Koszykowa 75, 00-662 Warsaw, Poland \\ ${ }^{b}$ Central Institute of Labor Protection, National Research Institute \\ Czerniakowska 16, 00-701 Warsaw, Poland
}

(Received April 17, 2003)

\begin{abstract}
In the paper thermodynamic properties of an artificial neural network are analyzed in a way analogous to spin glasses theory. Synaptic connections are calculated numerically according to the Hebb rule and their distribution is obtained for different characteristics of stored patterns. The phase diagrams and magnetization are established in dependence on the temperature of the network and the external field (threshold). It was showed that changing control parameters typical of artificial neural network (i.e. number of stored patterns and pattern bias level) one obtains the results similar to the Sherrington-Kirkpatrick model of spin glass.
\end{abstract}

PACS numbers: $75.10 . \mathrm{Hk}$

\section{Introduction}

The thermodynamics of the Hopfield model of artificial neural networks $(\mathrm{ANN})$ with synaptic connections resulting from a set of random patterns stored in the network and $T>0$ was investigated extensively since years (see e.g. [1-4]). In the majority of papers this analysis is based on the similarity of ANN to spin glasses. As it is well known, spin glasses are an example of spatially disordered magnetic systems in which ferromagnetic impurities are randomly distributed in the diamagnetic metal (e.g. CuMn, AuFe) and coupled by the long-range exchange interactions with the signs depending on the distance between magnetic atoms (see e.g. [5]). Many authors investigated spin glass systems, because of its interesting physical properties e.g. frustration, the lack of ergodicity etc. [6-13]. In the most 
popular model of spin glasses proposed by Sherrington and Kirkpatrick [6, 7] a set of the Ising spins $S_{i}(i=1,2,3, \ldots, N)$ is located in a periodic lattice. Spins are coupled by the infinite range exchange interactions $J_{i j}$ distributed with the Gaussian probabilities. Magnetic properties of such a system can be calculated using statistical physics with some special approximations e.g. replica method for calculation of free energy $[6,7]$. As a result, a magnetic phase diagram is found which shows the ranges of the stability of spin glass, ferromagnetic and paramagnetic phases in dependence on average value of exchange interactions $J_{0}$ and the temperature $T$.

Similar diagrams were found for neural networks. It was possible to determine the ranges of stability of neural network states with respect to the memory properties of the network. Such results were obtained for the first time by Amit et al. [14].

In the present work the Hopfield model of neural network is investigated numerically for the case of random, biased patterns stored in the network, which enables to compare the results with the results of the Sherrington-Kirkpatrick model of spin glass. Also the influence of external field $h_{\text {ext }}$ on the system is investigated. In contrast to other models we do not assume a priori a special distribution of connections parameters $J_{i j}$, but evaluate it numerically for every set of patterns initially stored in the network.

\section{The model}

In our model neural network consists of $N$ neurons $S_{i}= \pm 1$, which are updated synchronically according to the rule

$$
\begin{array}{lll}
S_{i}(t+1)=+1 \quad \text { with probability equal to } & \frac{1}{2}\left\{1+\tanh \left[\frac{h_{i}(t)-h_{\mathrm{ext}}}{T}\right]\right\}, \\
S_{i}(t+1)=-1 \quad \text { with probability equal to } & \frac{1}{2}\left\{1-\tanh \left[\frac{h_{i}(t)-h_{\mathrm{ext}}}{T}\right]\right\},
\end{array}
$$

where $h_{\text {ext }}$ is the external field and $h_{i}(t)$ is the local field given by

$$
h_{i}(t)=\sum_{j \neq i}^{N} J_{i j} S_{j}(t) .
$$

Synaptic connections $J_{i j}$ were calculated using the Hebb rule for the set of $p$ random patterns $\left\{\xi_{i}^{\mu}\right\}, \mu=1,2,3, \ldots, p$. In our computations the case of random unbiased and random biased patterns were used. On the basis of central limit theorem $J_{i j}$ are random variables with the Gaussian distribution, mean value $J$, and variance $\theta^{2}=p / N[15]:$

$$
P\left(J_{i j}\right)=\frac{1}{\theta \sqrt{2 \pi}} \exp \left[-\frac{\left(J_{i j}-J\right)^{2}}{2 \theta^{2}}\right] .
$$


The process of forming Gaussian distribution of synaptic connections when $p=20$ random and unbiased patterns is stored in the network with $N=100$ neurons is shown in Fig. 1. In this case the distribution of synaptic connections is symmetric and $J=0$. For the biased random patterns the synaptic connections are non-symmetric. Its form for the case of $p=1,5,10$, and 30 patterns, in which the probability $P$ of appearance of the value $S_{i}=+1$ of a neuron ranges from $P=0.5$ to $P=1$, stored in the network with $N=100$ neurons, is shown in Fig. 2. The dependence of the mean value of synaptic connections distribution on the value of probability $P$ is shown in Fig. 3 for the case of $p=20$ and $p=40$ stored patterns.
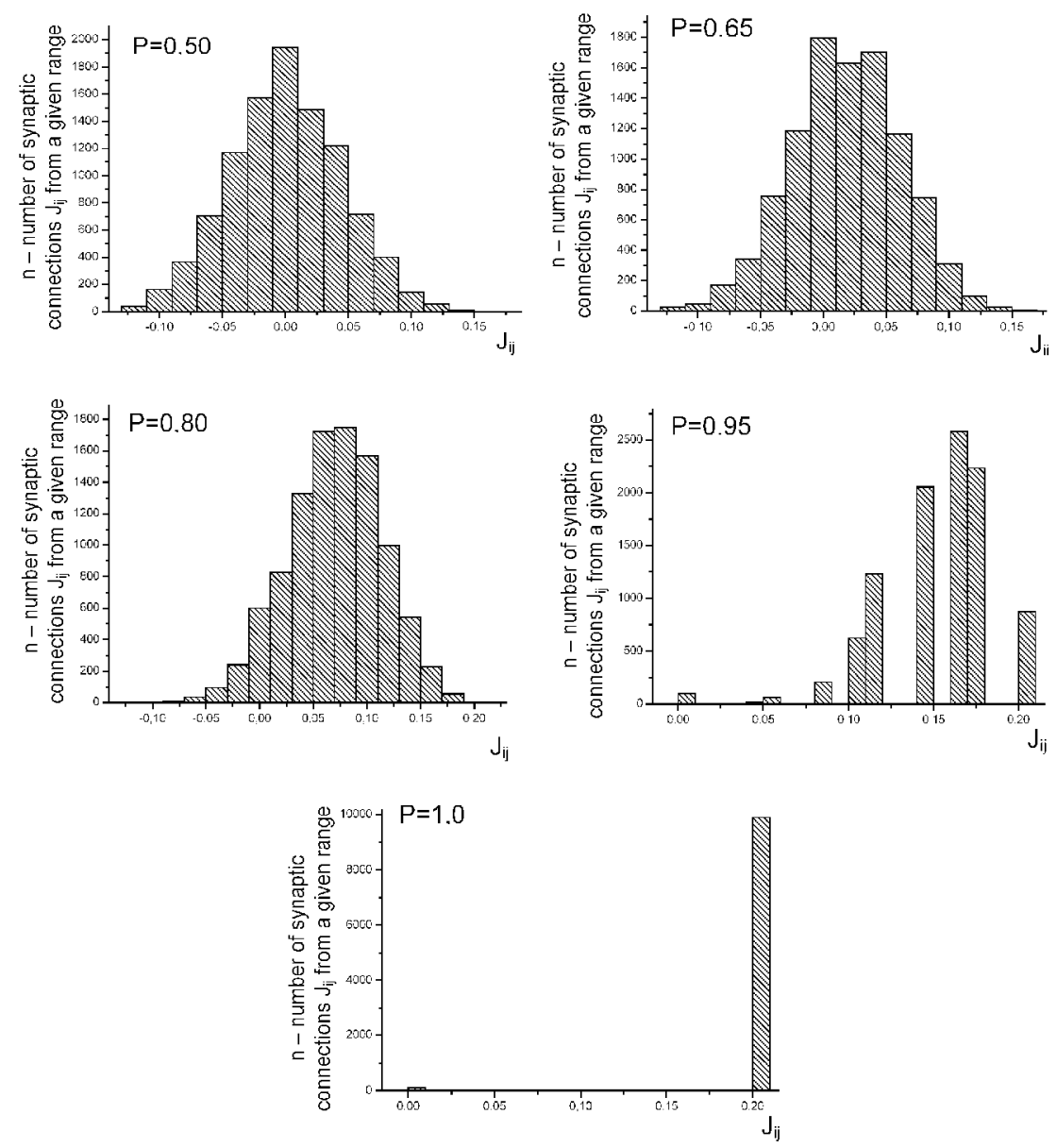

Fig. 1. Distribution of synaptic connections for $N=100$ and $p=20$ (number of random and unbiased patterns) and different values of parameter $P$ (mean value of $S$ for a stored pattern). 

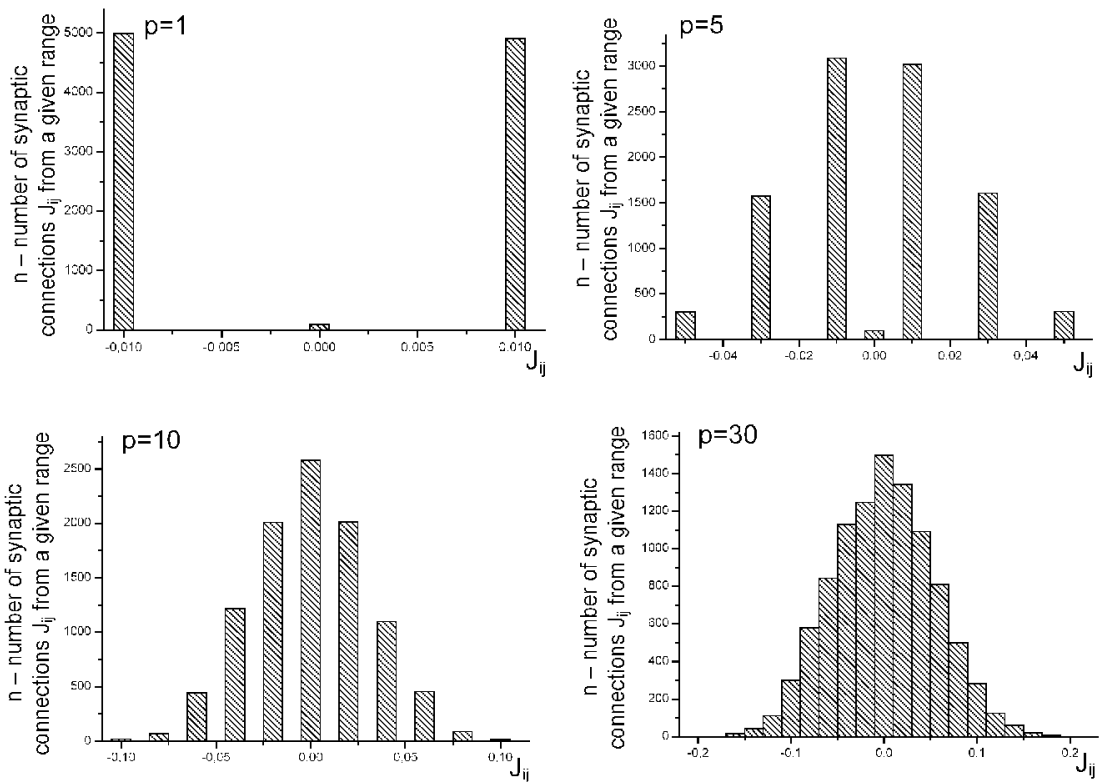

Fig. 2. Distribution of synaptic connections for $N=100, P=0.5$ and for $p=1,5,10$, and 30 .

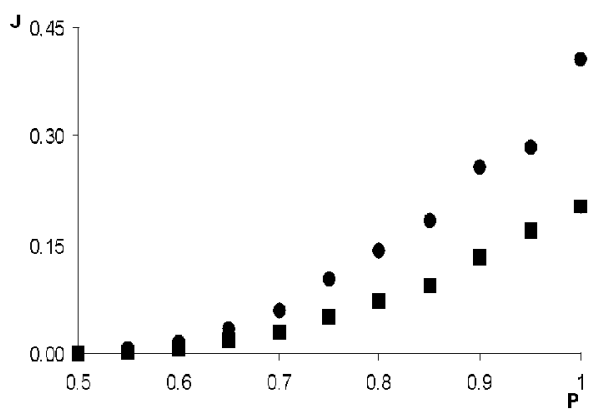

Fig. 3. Dependence of the mean value $J$ of $J_{i j}$ as function of the probability $P$ for two values of $p:-p=20 ; \bullet p=40$.

The phase diagram of the network under investigation was found by calculation of magnetization

$$
M(t)=\left\langle\frac{1}{N} \sum S_{i}(t)\right\rangle_{J},
$$

where \langle\rangle$_{J}$ means the averaging over different realizations of distribution of synaptic connections (equals in our computations to 100). Magnetization is non-zero in ferromagnetic phase and $M=0$ in spin glass and paramagnetic phase. In order to differ spin glass and paramagnetic phase observation of the order parameter $q$ is necessary. This quantity which shows the time correlation of the values of 
spins was differently defined, because of the non-ergodicity of spin glass systems $[6,7,16,17]$. In our calculations we used a definition

$$
q \equiv \lim _{t \rightarrow \infty}\left\langle S_{i}\left(t_{\mathrm{r}}\right) S_{i}\left(t-t_{\mathrm{r}}\right)\right\rangle_{\mathrm{J}}
$$

where $t_{\mathrm{r}}$ is the relaxation time of the network during the evolution, which was approximated individually for each run of computations. Random distributions of the initial states of neurons $S_{i}(0)$ as initial conditions for computations and synchronous dynamics were used.

\section{Results and discussion}

The magnetization as a function of temperature $M(T)$ for different values of probability $P$ (i.e. different bias of patterns) is shown in Fig. 4. From this figure it follows that in low temperatures the system is ferromagnetically ordered. When the temperature increases, the thermal fluctuations of neurons lead to decrease in magnetization. When the probability $P$ of excitation of each neuron increases, the decrease in $M(t)$ is slower, because the ordering influence of local fields, coming from other neurons, is greater. Consequently, the critical temperature $T_{\mathrm{c}}$, in which $M(t)$ reaches zero, increases with the probability $P$ (Fig. 5).

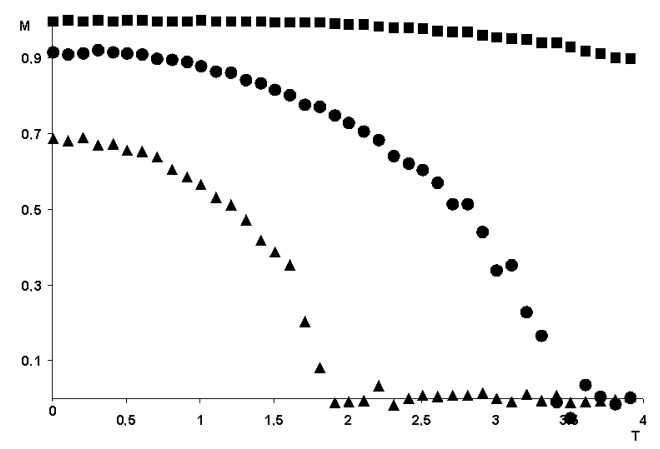

Fig. 4. Temperature dependence of magnetization $M$ for three values of $P(p=20)$ : full $\Delta P=0.65 ; \bullet P=0.75 ; \backsim P=0.90$.

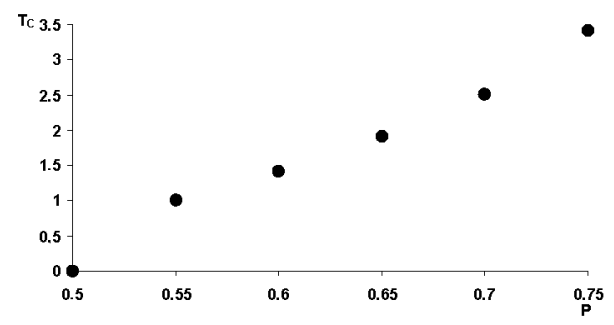

Fig. 5. Dependence of the critical temperature $T_{\mathrm{c}}$ on $P$. 


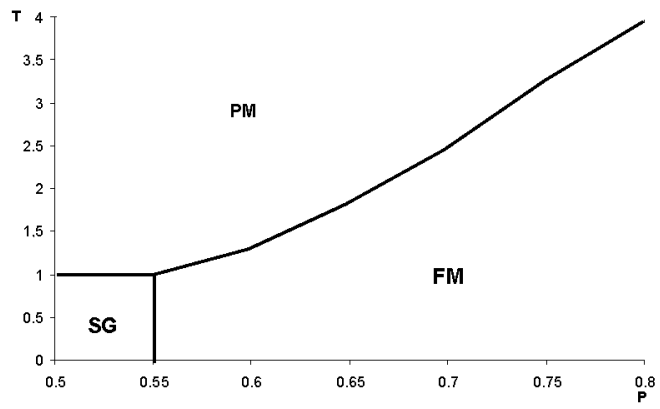

Fig. 6. Phase diagram $(P, T)$ for the network considered.

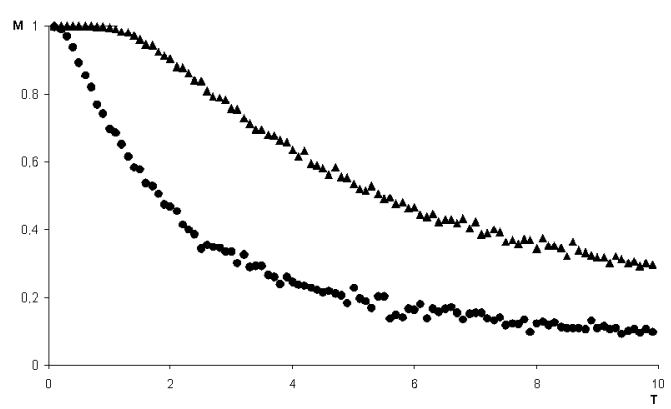

Fig. 7. Temperature dependence of $M$ on the external field (threshold) $h_{\text {ext }}$ : - $h_{\mathrm{ext}}=-1$; full $\Delta h_{\mathrm{ext}}=-2$.

Calculations of the parameters $M$ and $q$ (Eqs. (4), (5)) for the asymptotic behavior of the network in its time evolution for different temperatures and different values of probability $P$ allow to find phase diagram of the considered network (Fig. 6). It shows the ranges of stability of ferromagnetic $(M \neq 0, q \neq 0)$, spin glass $(M=0, q \neq 0)$ and paramagnetic phases $(M=0, q=0)$. It can be noticed that this phase diagram agrees rather well with the phase diagram obtained for the magnetic system by Sherrington and Kirkpatrick, as well as with later results [18].

Taking in our assumptions into account external field, it is interesting to interpret this parameter in the terms of the mean field theory applied to ANN. In the mean field theory the thermodynamical average of a spin equals to $\left\langle S_{i}\right\rangle=$ $\tanh \left(\beta\left\langle h_{i}\right\rangle\right)$. The mean value $\left\langle h_{i}\right\rangle$ of the field is equal to

$$
\left\langle h_{i}\right\rangle=\sum_{j=1}^{N} J_{i j}\left\langle S_{j}\right\rangle \quad(j \neq i) .
$$

If we assume the Hebb rule for $J_{i j}$, then the above relation may be rewritten in the following form: 


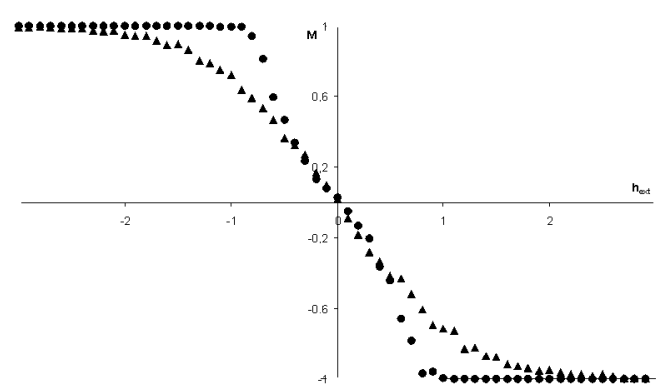

Fig. 8. Relation $M\left(h_{\text {ext }}\right)$ for different temperatures: $\bullet T=10^{-6}$; full $\Delta T=1$.

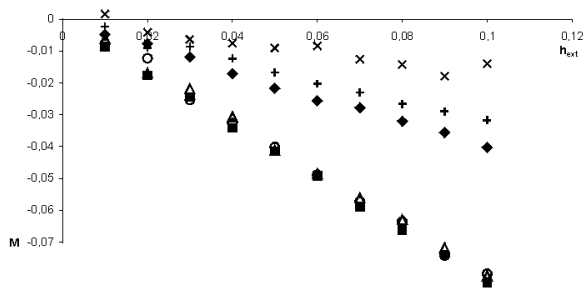

Fig. 9. Relation $M\left(h_{\text {ext }}\right)$ for $h_{\text {ext }} \rightarrow 0$ and different temperatures: - $T=0.01$; $\Delta T=0.5 ; \circ T=1 ;$ full $\diamond T=2.5 ;+T=3 ; \times T=6$.

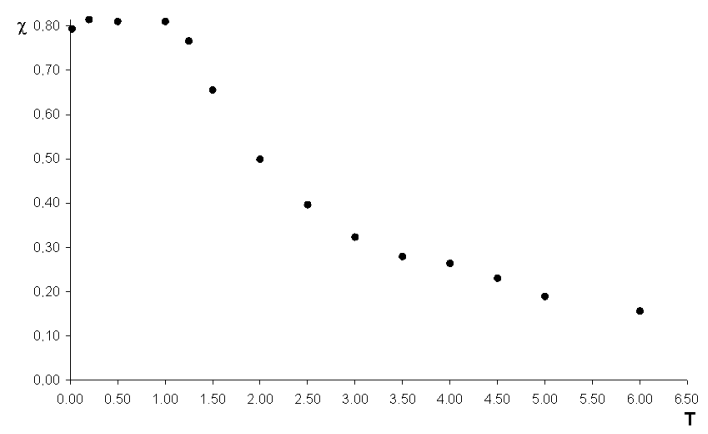

Fig. 10. The susceptibility of the network as function of temperature for vanishing external field.

$$
\left\langle h_{i}\right\rangle=\frac{1}{N} \sum_{\mu=1}^{p} \sum_{j=1}^{N} \xi_{i} \xi_{j}\left\langle S_{j}\right\rangle+h_{\mathrm{ext}},
$$

where $h_{\text {ext }}=-\frac{p}{N}\left\langle S_{i}\right\rangle$ has the meaning of a stabilizing factor arising from the stored patterns.

Influence of the external field $h_{\text {ext }}$ as an ordering factor can be observed at $M(T)$ curves (Fig. 7). Increase in external field causes the saturation even in low temperatures. On the other hand, when the temperature increases, magnetization does not fall to zero, and spin glass phase is not observed [8-10]. Competition 
between ordering influence of $h_{\text {ext }}$ and disordering influence of $T$ is seen in Fig. 8, where the magnetization as a function of $h_{\text {ext }}$ for different values of temperature is shown. The last two figures (Figs. 9,10) show the behavior of $M$ for very weak fields. There exists a critical value of $T$, above which the susceptibility roughly follows the Curie-Weiss law.

Concluding, it can be noticed that the thermodynamic properties of the Hopfield neural network are similar to the properties of spin glass magnetic system, which was a basis for statistical description of artificial neural networks $[1,2,14,15]$. The model used in this paper has a more general character and may easily be extended to more sophisticated networks.

\section{References}

[1] D.J. Amit, H. Gutfreund, H. Sompolinsky, Phys. Rev. A 32, 1007 (1985).

[2] D.J. Amit, H. Gutfreund, H. Sompolinsky, Ann. Phys. 173, 30 (1987).

[3] A. Crisanti, G. Paladin, H.J. Sommers, A. Vulpani, J. Phys. (France) 2, 1325 (1992).

[4] A. Theumann, Phys. Rev. E 53, 6361 (1996).

[5] A.P. Young, Spin Glasses and Random Fields, World Scientific, Singapore 1998.

[6] D. Sherrington, S. Kirkpatrick, Phys. Rev. Lett. 35, 1792 (1975).

[7] S. Kirkpatrick, D. Sherrington, Phys. Rev. B 17, 4384 (1978).

[8] J.R.L. Almeida, D.J. Thouless, J. Phys. Math. Gen. 11, 983 (1978).

[9] G. Parisi, J. Phys. A, Math. Gen. 13, 1887 (1980).

[10] F.T. Bantilan Jr., R.G. Palmer, J. Phys. F, Met. Phys. 11, 261 (1981).

[11] N.D. Mackenzie, A.P. Young, Phys. Rev. Lett. 49, 301 (1982).

[12] A. Houghton, A.P. Young, Phys. Rev. B 28, 2630 (1983).

[13] M. Mezard, G. Parisi, N. Sourlas, G. Toulouse, M. Virasoro, J. Phys. (France) 45, 843 (1984).

[14] D.J. Amit, H. Gutfreund, H. Sompolinsky, Phys. Rev. A 32, 1007 (1985).

[15] D.J. Amit, Modeling Brain Functions, Cambridge Univ. Press, Cambridge 1989.

[16] S.F. Edwards, P.W. Anderson, J. Phys. F, Met. Phys. 5, 965 (1975).

[17] G. Parisi, Phys. Rev. Lett. 50, 1946 (1983).

[18] K.P. Wilson, D.J. Goosens, Acta Phys. Pol. A 97, 983 (2000). 